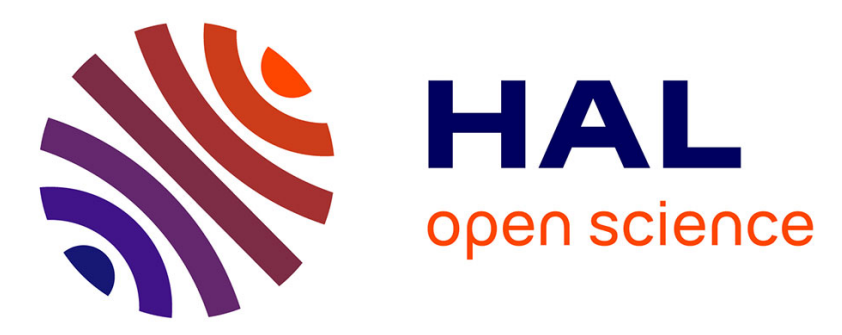

\title{
Influence of diffuse damage on the water vapour permeability of fibre reinforced mortar
}

Simon Rouchier, Geneviève Foray, Monika Woloszyn, Jean-Jacques Roux

\section{To cite this version:}

Simon Rouchier, Geneviève Foray, Monika Woloszyn, Jean-Jacques Roux. Influence of diffuse damage on the water vapour permeability of fibre reinforced mortar. Transport in Porous Media, 2012, 93, pp.543-559. 10.1007/s11242-012-9967-2 . hal-00761767

\section{HAL Id: hal-00761767 https://hal.science/hal-00761767}

Submitted on 6 Dec 2012

HAL is a multi-disciplinary open access archive for the deposit and dissemination of scientific research documents, whether they are published or not. The documents may come from teaching and research institutions in France or abroad, or from public or private research centers.
L'archive ouverte pluridisciplinaire HAL, est destinée au dépôt et à la diffusion de documents scientifiques de niveau recherche, publiés ou non, émanant des établissements d'enseignement et de recherche français ou étrangers, des laboratoires publics ou privés. 


\title{
Influence of diffuse damage on the water vapour permeability of fibre reinforced mortar
}

\author{
Simon Rouchier*1 ${ }^{*}$ Geneviève Foray ${ }^{2}$, Monika Woloszyn $^{3}$, and Jean-Jacques Roux ${ }^{4}$ \\ ${ }^{1}$ CETHIL CNRS UMR5008, MATEIS CNRS UMR5510, INSA-Lyon, Université Lyon 1, F-69621 Villeurbanne, France \\ 2 INSA-Lyon, MATEIS CNRS UMR5510, F-69621 Villeurbanne, France \\ ${ }^{3}$ LOCIE, CNRS-UMR5271, Université de Savoie, Campus Scientifique, Savoie Technolac, 73376 Le Bourget-du-Lac Cedex, France \\ ${ }^{4}$ CETHIL, CNRS UMR5008, INSA-Lyon, Université Lyon 1, Bât. S. Carnot, 9 Rue de la Physique, F-69621 Villeurbanne, France
}

Postprint: Rouchier S., Foray G., Woloszyn M., Roux J.-J., 2012. Influence of diffuse damage on the water vapour permeability of fibre reinforced mortar, Transport in Porous Media, vol. 93, pp. 543-559. DOI 10.1007/s11242012-9967-2

\begin{abstract}
The study of moisture transfer inside building materials is an important issue in building physics. The hygric characterization of such materials has become a common practice for the estimation of the hygrothermal performance of buildings. However, their aging caused by mechanical loading and environmental factors inevitably affects their permeability to moisture ingress, and the knowledge of how this permeability is affected by damage and cracks is still incomplete.

The effects of diffuse damage caused by mechanical loading on the water vapour permeability of fibre-reinforced mortar were studied. A full experimental setup is presented including observation of the porous structure, mechanical, and hygric characterization. Uniaxial tensile loading was applied on prismatic samples while their damage level was measured. Then, the moisture content of damaged and undamaged samples was monitored during variations of ambient relative humidity. Two numerical methods are presented and used for the comparison of the water vapour permeability of multiple samples presenting various levels of damage. By this methodology, diffuse damage caused by mechanical loading is shown to have an impact on the water vapour transfer inside the material.
\end{abstract}

Keywords damaged porous media; moisture transfer; water vapour permeability; diffuse damage

\section{Introduction}

The study of moisture transfer inside building materials is an important issue in building physics, as it influences the durability and sustainability of structures, the health and comfort of the residents and the thermal performance of the inner and outer walls. Owing to the necessary reduction in energy consumptions, new thermal insulation systems will need to prove their efficiency over their life span.

Coupled heat and moisture simulations prior to a building construction have become an important part of its design and a condition for its energetic efficiency. Since the first questionings on the subject, various methods were developed for the experimental determination of the equilibrium moisture content and permeability of construction materials. However, the material description in simulation tools is often based on hypothesis such as

*Electronic address: simon.rouchier@insa-lyon.fr $;$ Corresponding author 
anisotropy and constant transfer properties of the materials. While these assumptions could be accurate at the time of construction of a building, they could be a strong source of calculation errors as the building ages and its components start to present various forms of degradation. Fractures in cementitious materials can be caused by various phenomena: compressive and tensile mechanical loading and fatigue phenomena causing progressive microstructural dislocations [Elaqra et al, 2007], cyclic expansion of the material due to thermal cycles, degradation caused by sorption and desorption cycles, transport of reacting chemicals through water such as chloride ions. Microcracking first occurs during the maturation and hydration processes in Portland cement as a result of drying shrinkage [Bažant and Raftshol, 1982]. As a consequence, defects are already distributed at early age in concrete or mortar and represent a base for further cracking.

The focus here is on mechanically induced damage mechanisms and their impact on water vapour permeability. Building material durability studies tend to describe the degradation of semi-brittle materials such as concrete in two phases [Mazars and Pijaudier-Cabot, 1996] : damage mechanics [Bažant and Oh, 1983] for the description of diffuse microcracking and fracture mechanics for the growth of a macroscopic crack subsequent to a strainsoftening behaviour. In both cases, cracks have been shown to have a significant impact on transport processes in concrete [Aldea et al, 1999, Wang et al].

Depending on the nature and geometry of the crack network, several approaches have been developed for flow modelling in fractured porous media [Berkowitz, 2002]. Discrete fracture models, suited for macroscopic fractures or close-range modelling of cracks, allow taking into account detailed transport phenomena and moisture exchange between the porous medium and the fractures. Some methods allow simulating the effects of moisture on the evolution of crack opening and the mechanical behaviour of porous materials [Roels et al, 2006, Réthoré et al, 2007]. However, such models require an exhaustive material description and mechanical characterization prior to flow simulations.

Equivalent continuum models on the other hand can be suited for damaged building components with a high number of well-distributed microcracks. Recent studies [Pijaudier-Cabot et al, 2009] have shown the consequences of diffuse micro-cracking on the intrinsic permeability of concrete during mechanical loading or after unloading. The liquid and gaseous conductivity of cementitious materials can be significantly increased before the formation of a macroscopic fracture. However, moisture transport in building materials is not only caused by the displacement of a continuous liquid phase which can appear in case of rain, but is also caused by water vapour gradients and sorption processes in the hygroscopic range. The complete understanding of the influence of damage on these processes requires experimental characterization and numerical modelling methods.

A complete experimental setup starting from material elaboration up to mechanical loading and hygric characterization has been developed. Coupled with appropriate numerical tools, this methodology allowed estimating the effects of damage on the water vapour transfer properties of a glass fibre-reinforced mortar. This article starts with the outline of the methodology. Two methods are then explained for the estimation of hygric transport properties based on transient measurements of mass uptake in the mortar samples. The results are then displayed, and finite-volume simulations are shown to give an estimate on the possible consequences of diffuse damage on the dynamic hygric behaviour of a building component.

\section{Methodology}

The experimental setup elaborated in order to investigate the effects of diffuse cracking on the water vapour permeability of mortar is first described in this section.

\subsection{Material}

The material used for all tests is a commercial formulation (Lafarge, MAITE monocomposant) used for external thermal insulation composite systems [Chalencon et al, 2010]. It is a Portland cement mortar including dry redispersable polymer systems with a water to dry material weight ratio of 0.16 and reinforced with $1 \%$ weight of glass fibres. While cement particles are dissolved and hydrated, polymers are dispersed in the liquid phase, and form a continuous solid network with the formed hydrates and the unreacted cement or fillers. Earlier studies 


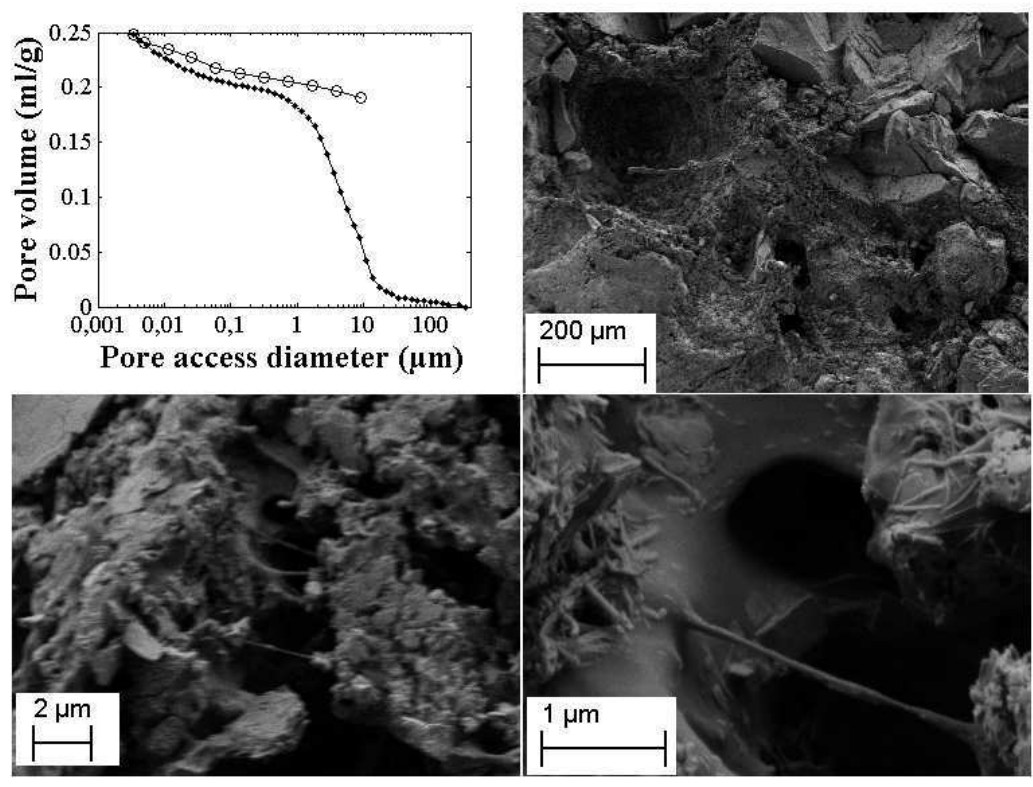

Figure 1: Upper-left image: pore intrusion (dots) and extrusion (circles) diameter distributions measured by mercury intrusion porosimetry. Other three images: SEM observations of the mortar including dispersed polymers

[Chalencon, 2010] proved that during tensile loading, the material shows a non-brittle behaviour, developing progressive diffuse cracking before the formation of a main macroscopic fracture. The sand and cement were mixed with water for a minute before incorporation of the glass fibres and two more minutes of mixing. The paste was then moulded into prismatic samples of $500 \mathrm{~g}$ and approximately $300 \times 100 \times 10 \mathrm{~mm}$, and kept two days at a $90 \%$ relative humidity to prevent excessive water loss of the cement paste. The samples were then unmoulded and kept for 21 days of maturation at 50\%RH: the mechanical behaviour of fibre-reinforced mortar resulting from this maturation procedure are known [Chalencon et al, 2010].

After maturation, and prior to its mechanical and hygric characterization, the porous structure of the material was observed by mercury intrusion porosimetry (MIP) and scanning electron microscopy (SEM), as shown on Fig. 1. The material has an open porosity of approximately $40 \%$ and an average density of $1450 \mathrm{~kg} \cdot \mathrm{m}^{-3}$.

Fig. 1 shows the mercury intrusion profile (below) with a pressure up to $400 \mathrm{MPa}$, accessing pore diameters of down to $3 \mathrm{~nm}$. The upper curve is the extrusion profile from this point, showing a hysteresis behaviour. As shown by the MIP profile, most of the pores are accessible by cavities and channels between 1 and $10 \mu \mathrm{m}$ of diameter. An important part of the pore volume is also present at smaller scales, as the slope of the intrusion profile is still important near the limit of mercury porosimetry. SEM images have confirmed the multiscale nature of the porosity: $200 \mu \mathrm{m}$ circular pores, shown on the upper-right SEM image, are connected by channels in the range of 1 to $10 \mu \mathrm{m}$ diameter, shown on the lower images, and the hydrates include nano-porosities.

All results presented in Sect. 4 concern three series of 16 samples. Since the conditions of sample manufacturing, ambient humidity and temperature in the laboratory can differ from one day to another, all comparisons of samples, in terms of hygric or mechanical properties, were made between samples of the same production series. This was to make sure that the measured differences in permeability were not due to outside factors. 

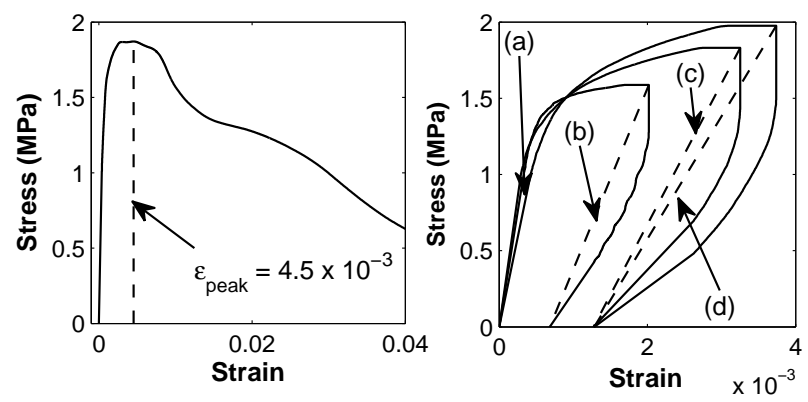

Figure 2: Stress-strain profiles on a full tensile loading (left) and on three tests interrupted before peak strain (right): (a) $E_{0}=2.75 \mathrm{GPa}$, (b) $E_{e}=1170 \mathrm{MPa}$, (c) $E_{e}=859 \mathrm{MPa}$, (d) $E_{e}=782 \mathrm{MPa}$

\subsection{Mechanical characterization}

After maturation, the prismatic samples were damaged by tensile loading beyond their limit of elasticity, using a $5 \mathrm{kN}$ force cell imposing a constant displacement speed of $1 \mathrm{~mm} / \mathrm{min}$. As a first step, the full tensile response of the mortar was observed with a full mechanical loading for the estimation of the peak strain (see left side of Fig. 2). Repeated observations, earlier confirmed by stereocorrelation [Chalencon, 2010] showed that a macroscopic fracture initiates during the strain-softening phase subsequent to the peak strain $\epsilon_{\text {peak }}$.

In order to apply diffuse microscopic damage on the samples before hygric characterization, tensile loading on the tested samples was manually interrupted before the peak strain, as shown on the right side of Fig. 2 . The diffuse damage induced by this mechanical loading was characterized by a single scalar value $D$, defined as the relative change in their Young modulus [Mazars and Pijaudier-Cabot, 1989]:

$$
D=1-\frac{E_{e}}{E_{0}}
$$

where $E_{0}$ is the initial elastic modulus of a sample and $E_{e}$ is its effective modulus after unloading, as shown with dotted lines on Fig. 2. $D$ spans from 0 for undamaged materials to 1 for fully fractured samples, although this value is not reached within the frame of this study.

Among the tested series of samples, the value of the scalar damages reached with tensile loading span from 0 (samples kept intact) to 0.7 (before peak stress), higher damage being difficult to reach without causing a macroscopic crack initiation, i.e. the appearance of a localized crack of over $100 \mu \mathrm{m}$ aperture, in which case an equivalent permeability cannot be defined over the surface of the sample. After unloading, the central part of each plate was used for hygric characterization in a climatic chamber.

\subsection{Hygric characterization}

Samples of dimensions $100 \times 100 \times 10 \mathrm{~mm}$ were extracted from the plates after unloading and insulated to moisture on all faces but one with aluminium tape, as shown on Fig. 3. Only the upper face was left unsealed, so that mass transfer could be considered unidimensional in all tests. The samples were then placed on up to eight weighing systems inside a climatic chamber, continually recording their mass without the need to take them out of the chamber, thus avoiding disturbances in the ambient humidity level. The systems can measure mass with a precision of $10^{-3} \mathrm{~g}$, and were set to provide averaged values of 20 consecutive weighings every $10 \mathrm{~s}$ to reduce noise effects in the results. The inside of the chamber is ventilated by a fan which ensures a homogeneous distribution of $\mathrm{RH}$ and temperature. This was confirmed by distributing probes prior to the test as to test the ability of the chamber to recreate a stepwise change in relative humidity in isothermal conditions. The average recorded time for the humidity to stabilize after a change in the set point was $14 \mathrm{~min}$. The maximal deviation of temperature from its expected value was $0.4 \mathrm{~K}$.

The hygric characterization of the fibre-reinforced mortar was run inside the climatic chamber following two different pre-programmed variations of relative humidity as shown on Fig. 4. Each of these programs served as 


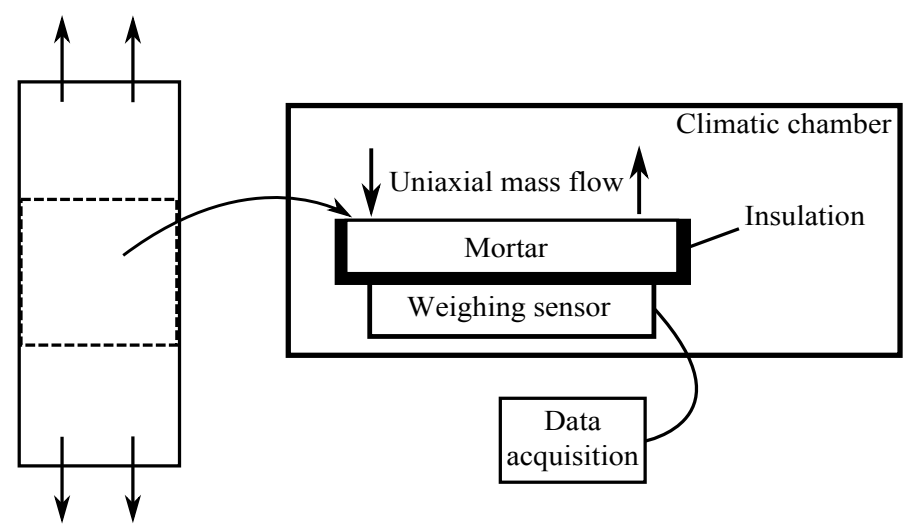

Figure 3: Sketch of the sample extraction from the loaded plates and Hydrodyn experimental setup developed in MATEIS
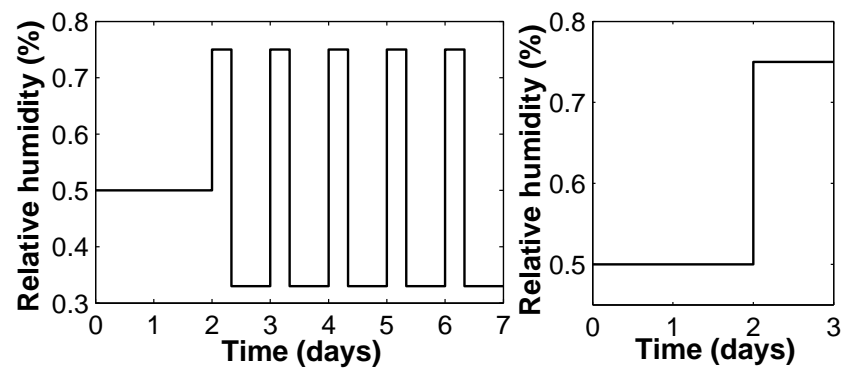

Figure 4: Relative humidity programs inside the climatic chamber, respectively method 1 (left) and 2 (right)

basis for the estimation of the water vapour permeability of the material, as explained below. In both cases, a constant humidity of $50 \%$ is imposed during the first $48 \mathrm{~h}$, in order to reduce the mass uptake rate before the start of the stepwise variations. Although equilibrium is not strictly reached, the mass variations at the end of these 48 $\mathrm{h}$ are negligible in comparison to the uptake rate after a sudden change in hygric boundary conditions.

The first program followed the Nordtest protocol [Rode, 2003], which was originally proposed for the definition and experimental characterization of the moisture buffering capacity of building materials. The purpose was to produce successions of high and low humidity phases, similar to either outdoor or indoor conditions in buildings, and to observe the rate and amplitude of the variations of moisture content in the materials. In this case, the chamber imposed cycles consisting of a high humidity step ( $8 \mathrm{~h}$ at $75 \%$ relative humidity) and a longer low humidity step ( $16 \mathrm{~h}$ at $33 \% \mathrm{RH}$ ) at a constant temperature of $23^{\circ} \mathrm{C}$ (see Fig. 4). These $24 \mathrm{~h}$ sequences were repeated over several days, until two successive days of measurement presented the same variations of moisture content. The recorded mass uptake in a mortar sample during steps of higher humidity was used as basis for the estimation of its permeability, as described in Sect. 3.2.1. This approach also gave a direct outline of the behaviour of the material in ambient humidity conditions approaching in situ building conditions.

The second program shown on Fig. 4 was a single step from 50 to $75 \%$ relative humidity. The recorded mass uptake as a response to this step was interpreted following Sect. 3.2.2. This program allows estimating the permeability, and moreover gave a theoretical value of the equilibrium water content and the surface transfer coefficient, as explained below.

Both methods are based on the assumption that the equilibrium moisture content of the material was not affected by damage. In order to confirm this hypothesis, the total mass uptake from $50 \% \mathrm{RH}$ to $75 \% \mathrm{RH}$ was measured in intact and damaged samples, in addition to the measurements of water vapour permeability. Hysteresis effects between the sorption and desorption profiles were not considered, since all permeability estimations were based 


\section{Methods for the estimation of moisture permeability}

\subsection{Isothermal moisture transport equation}

The modelling of transfer through porous building materials for the study of their hygrothermal performances runs through Heat, Air and Moisture (HAM) transfer models which are based on the resolution of up to three coupled transfer equations, respectively, for heat, air and moisture balance [Hagentoft 2001]. Air flow occurs through building components in case of atmospheric pressure fluctuations or differences between the indoor and outdoor pressures, and can have a strong influence on heat and moisture transfer. This is shown by additional convective terms in their respective transport equations. Whereas air pressure can have a significant influence on the drying processes of concrete [Mainguy et al, 2001], the characterization of moisture transport properties of a material require the hypothesis of a constant atmospheric pressure, which is often the case in the modelling of coupled heat and mass transfer [Janssen et al, 2007] or in the modelling of material properties [Scheffler and Plagge, 2010].

The conservation equation for the water content $w$ inside a building component includes terms for moisture transport through vapour diffusion in the gaseous phase $\mathbf{g}_{v}$ and through the displacement of a liquid phase $\mathbf{g}_{l}$, which are usually expressed separately:

$$
\frac{\partial w}{\partial t}=-\nabla \cdot\left(\mathbf{g}_{v}+\mathbf{g}_{l}\right)
$$

Under the hypothesis that air transfer does not occur, the water vapour transfer is driven by a gradient of water vapour pressure $p_{v}$, and liquid water transfer is driven by a gradient of capillary pressure $p_{c}$.

$$
\begin{aligned}
& \mathbf{g}_{v}=-\delta_{p} \nabla p_{v} \\
& \mathbf{g}_{l}=-K_{l} \nabla p_{c}
\end{aligned}
$$

where $\delta_{p}$ and $K_{l}$ are respectively the water vapour and liquid permeabilities of the material, expressed in unit $\left[\mathrm{kg} \cdot \mathrm{m}^{-1} \cdot \mathrm{s}^{-1} \cdot \mathrm{Pa}^{-1}\right]$ or (s). The term $\delta_{p}$ is sometimes depicted in the literature as the water vapour diffusivity of the material, as it is related to the diffusivity of water in air $D_{v, a}$ as follows:

$$
\delta_{p}=\frac{1}{\mu} \frac{D_{v, a}}{R T}
$$

where $R$ is the specific gas constant of water vapour, $T$ the temperature and $\mu$ the so-called resistance factor of the material, which describes the rate of diffusion of water in air through the porous network. Because of dimensionality arguments, $\delta_{p}$ is here referred to as water vapour permeability. Water vapour transfer is prevailing over liquid transfer in the lower humidity range, in the absence of a continuous liquid phase in the porous network. Liquid transfer is prevailing in the opposite case, for instance when a building component is in contact with wind-driven rain, or for the study of the drying processes of concrete [Mainguy et al, 2001, Baroghel-Bouny et al, 1999].

To model both phases as driven by the same potential (usually $p_{c}$ ) for numerical applications, the Kelvin formula can be used to relate both pressures, although the formulation of their spatial and temporal derivatives leads to additional coupling terms in the equation [Janssen et al, 2007]. The estimation of the liquid permeability $K_{l}$ of non-damaged materials can be achieved through network models [Xu et al, 1997, Xu et al, 1997, Carmeliet et al, 1999], which allow to extract the intrinsic or relative permeability of the material from the moisture retention curve or from experimental characterization methods of the material microstructure such as mercury intrusion porosimetry.

The vapour exchange at the surface of a sample is described by a convective mass transfer coefficient $h_{p}$ :

$$
\delta_{p}\left(\nabla p_{v} \cdot \mathbf{n}\right)=h_{p}\left(p_{v, \text { ext }}-p_{v}\right)
$$

where $p_{v, \text { ext }}$ is the ambient water vapour pressure and $\mathbf{n}$ the normal vector of the surface. The convective coefficient $h_{p}$ characterizes the boundary layer at the surface of the sample and is a function of multiple parameters 
such as the moisture concentration, the air velocity or the presence of turbulence. Its accurate modelling is usually challenging, and a closer look on methods for its experimental measurement can be found in [Kwiatkowski, 2009]. The methods presented below for the estimation of $\delta_{p}$ deal with this matter as follows: in method 1 (square root of time), the surface layer resistance is neglected, whereas the value of $h_{p}$ is estimated by method 2 (analytic resolution of the conservation equation).

\subsection{Water vapour permeability from experimental measurements}

The focus here is on the water transfer in the gaseous phase, which largely prevails in the hygroscopic humidity range considered in this study. Two different methodologies are presented for the derivation of the vapour permeability from the mass uptake measurements of the samples placed in a climatic chamber with stepwise changes in relative humidity.

Various methods have already been proposed in the literature for the derivation of moisture permeability of cementitious materials from stepwise sorptive or desorptive measurements [Garbalinska, 2006, Anderberg and Wadsö, 2008]. Two methods were implemented here as they appropriately cope with the experimental procedure described above. The first method presented here consisted in relating the permeability $\delta_{p}$ to the slope of the adsorption rate after a stepwise change in ambient relative humidity, plotted versus square root of time. It allowed comparison of several samples placed without any need of longer experimental times. The second method was based on an analytical resolution of the simplified moisture transport equation.

\subsubsection{Method 1: square root of time}

The notion of moisture buffer value (MBV) of a building material was introduced as part of the Nordtest project [Rode, 2003] which aimed at finding an appropriate measure for the adsorption and release of moisture by hygroscopic materials subjected to daily environmental fluctuations. The MBV is connected to the moisture effusivity $b_{m}$, which is an indicator of the rate of moisture intrusion after a given change in the boundary condition.

$$
b_{m}=\sqrt{\delta_{p} \frac{\partial w}{\partial p_{\nu}}}=\sqrt{\frac{\delta_{p} \xi}{p_{s a t}}}
$$

where $\xi=\partial w / \partial \phi$ is the slope of the sorption isotherm, or moisture capacity ( $\phi$ being the relative humidity), and $p_{\text {sat }}$ is the saturation pressure of water vapour in air.

Shortly after a stepwise change in ambient relative humidity, a fairly accurate approximation is to consider the instantaneous mass uptake rate in a specimen $g_{m}$ as proportional to the ratio of effusivity to the square root of time [Rode, 2003]:

$$
g_{m} \propto \frac{b_{m}}{\sqrt{t}}
$$

where $t$ is the elapsed time since the change in the moisture boundary condition. Equation 8 holds as long as the moisture penetration depth does not exceed the sample thickness. In the present study, time steps of $8 \mathrm{~h}$ are performed and this assumption is considered valid in regards to the expected value of the permeability and the sample thickness.

Equation 8 is made possible under the assumption that relatively small humidity steps are considered, and that the moisture resistance caused by the boundary layer at the surface of the material is small in comparison to the water vapour resistance of the material itself. Under these hypotheses, it is possible to find a relation of proportionality between the measured mass uptake $\Delta m$ of a sample during a humidity timestep after a time $t$ and its permeability $\delta_{p}$ :

$$
\frac{\Delta m}{S}=\int_{0}^{t} g_{m} \mathrm{~d} \tau \propto \sqrt{\frac{\delta_{p} \xi}{p_{s a t}}} \sqrt{t}
$$

where $S$ is the exposed surface of the sample. This surface of contact with the environment is prevailing over the volume of the sample for the mass uptake rate, as long as the sample thickness exceeds the moisture penetration depth for the fluctuations at stake. Equation 9 states a relation of proportionality: absolute values of permeability 
cannot be estimated by this procedure but relative variations could be measured between several samples which were subjected to the same humidity steps. If we express the ratio of expression 9 for two material specimens represented by subscripts 1 and 2 , the relation yields:

$$
\frac{\delta_{p, 1}}{\delta_{p, 2}} \frac{\xi_{1}}{\xi_{2}}=\left(\frac{\Delta m_{1}}{\Delta m_{2}} \frac{S_{2}}{S_{1}}\right)^{2}
$$

It is therefore possible to estimate the relative evolution of the permeability with the development of damage compared to its value for the intact material, under the assumption that the slope of the sorption isotherm $\xi$ is not influenced by the presence of fractures, which is relevant with the usual observation that cracks do not participate in the equilibrium moisture content of a material, especially one with relatively high porosity as is the case in this study. In method 1, the permeability is computed using Eq. 10 after the mass uptake recorded during each period of high humidity of the Nordtest protocol, assuming that the moisture content distribution is close to homogeneous at the end of a low humidity step. This method however only allows estimating ratios between permeabilities of several samples, rather than their absolute values.

\subsubsection{Method 2: analytic resolution of the transport equation}

A second procedure is proposed for the derivation of $\delta_{p}$ from mass uptake measurements, which theoretically also allows for a simultaneous estimation of all three parameters needed for mass transfer simulations: permeability $\delta_{p}$, moisture capacity $\xi$ and convective mass transfer coefficient at the surface of the sample $h_{p}$. The method is based on an analytical resolution of the moisture transport equation under following assumptions:

- the moisture transfer is one-dimensional

- the permeability and moisture capacity are constant in the observed humidity range

- coupling terms induced by air and heat transfer are neglected

The second hypothesis restricts the procedure to a limited interval of relative humidity, which must also not exceed a certain value above which the sorption isotherm becomes strongly non-linear. In this case, this interval will be $50 \%-75 \%$. As a consequence of these simplifications, the Eq. 2 becomes:

$$
\frac{\partial w}{\partial t}=\delta_{p} \frac{\partial p_{v}}{\partial w} \frac{\partial^{2} w}{\partial x^{2}}=\delta_{p} \frac{p_{s a t}}{\xi} \frac{\partial^{2} w}{\partial x^{2}},
$$

Equation 11 is similar to one-dimensional heat transfer through solids and can be analytically solved [Polifke and Kopitz, 2005, Garbalinska et al, 2010] for a sample of thickness $L$, exposed on one surface and insulated on the other, and initially placed at a uniform moisture content $w_{0}$ :

$$
\begin{gathered}
m=\int_{0}^{L}\left(w(x, t)-w_{0}\right) \mathrm{d} x \\
m=L \xi \Delta \phi\left[1-\sum_{k=1}^{\infty} \frac{2 \sin ^{2} a_{k}}{a_{k}\left(a_{k}+\sin a_{k} \cos a_{k}\right)} \exp \left(-a_{k}^{2} \frac{\delta_{p}}{\xi} \frac{p_{\text {sat }} t}{L^{2}}\right)\right]
\end{gathered}
$$

where $m$ is the mass increase by unit surface of the sample since the beginning of the humidity step $\Delta \phi$. This formulation is summed over a series of increasing numbers $a_{k}$ defined after the hygric Biot number $\mathrm{Bi}_{h}$. This number is defined similarly to its thermal equivalent (see for instance [Polifke and Kopitz, 2005]) as a measure of the comparative influence of the surface transfer resistance to diffusion phenomena across the sample of width $L$ :

$$
\begin{gathered}
\frac{\mathrm{Bi}_{h}}{a_{k}}=\tan a_{k} \\
\mathrm{Bi}_{h}=\frac{h_{p} L}{\delta_{p}}
\end{gathered}
$$


Table 1: Average value $\overline{\Delta w}$ and standard deviation $\widetilde{\Delta w}$ of the equilibrium moisture content variation from $50 \% \mathrm{RH}$ to $75 \% \mathrm{RH}$

\begin{tabular}{|ccc|}
\hline & $\overline{\Delta w}\left[\mathrm{~kg} \cdot \mathrm{m}^{-3}\right]$ & $\widetilde{\Delta w}\left[\mathrm{~kg} \cdot \mathrm{m}^{-3}\right]$ \\
$D=0$ (8 samples) & 6.27 & 0.63 \\
$D>0$ (6 samples) & 6.12 & 0.47 \\
\hline
\end{tabular}

The $a_{k}$ numbers are of decreasing influence on the result of the expression 13 and a sufficiently accurate result can be reached by considering only the first 5-10 terms of the series, depending on the material.

A Levenberg-Marquardt algorithm [Marquardt, 1963] is then applied for the estimation of the transport properties by correlating the non-linear Eq. 13 with mass uptake measurements by the least-squares method. This algorithm is based on the iterative resolution of the following system of equations:

$$
\left[\mathbf{J}^{t} \mathbf{J}\right]_{n} \mathbf{u}_{n+1}=\mathbf{J}_{n}^{t}\left[m_{\exp , i}-m\left(\mathbf{u}_{n}, t_{i}\right)\right]
$$

where the subscript $n$ indicates the iteration, $m_{\text {exp }, i}$ is a series of experimental measurements at the time coordinates $t_{i}, \mathbf{u}$ is the vector of the parameters $\left(\delta_{p}, \xi, h_{p}\right)$ and $\mathbf{J}$ is the matrix of the derivatives of $m$ with respect to $u$ :

$$
\mathbf{J}_{i, j}=\frac{\partial m\left(\mathbf{u}, t_{i}\right)}{\partial u_{j}}
$$

The expressions of $\partial m / \partial \delta_{p}, \partial m / \partial h_{p}$ and $\partial m / \partial \xi$ are required in this expression and can all be derived analytically from Eq. 13.

This procedure requires a sufficient input of mass measurements $m_{\text {exp } i}$, preferably evenly spaced along the humidity step. It theoretically allows the calculation of all parameters influencing the water uptake rate of the specimen, but might lack of accuracy when attempting a simultaneous estimation of all three, due to the approximations. These matters will be addressed in Sect. 4.2.2.

\section{Results and discussion}

Each of the two methodologies presented in Sect. 3.2 was applied with its corresponding humidity program in order to simultaneously measure the permeability of sample series. The advantages of each method are compared and summed up as a conclusion.

\subsection{Equilibrium moisture content}

To confirm the assumption under which fractures have a negligible impact on the sorption isotherm of fibrereinforced mortar, the equilibrium weight of intact and damaged specimen was measured at two humidity points (50 and $75 \% \mathrm{RH}$ ). The samples were first put in a stable $50 \% \mathrm{RH}$ environment until complete equilibrium was reached (defined as two identical consecutive weighings at $48 \mathrm{~h}$ time interval). The same measurements were then performed at $75 \% \mathrm{RH}$. The results are displayed in Table 1.

Although this data did not represent a full characterization of the sorption isotherm, they did confirmed the hypothesis made. Indeed, the porosity of the studied material being relatively high, its specific area was globally not impacted by smeared cracks. These measurements also allow an other assumption: the total mass uptake under $75 \% \mathrm{RH}$ is quite low and allowed assuming that no continuous liquid phase has appeared in the porous network, thus validating the present methodology for the estimation of the water vapour permeability.

\subsection{Water vapour permeability}

\subsubsection{Method 1: square root of time}

Under the hypotheses formulated in Sect. 3.2.1, the permeability of the samples placed in the climatic chamber is proportional to the square of the slope of mass uptake versus square root of time after a stepwise rise in ambient 


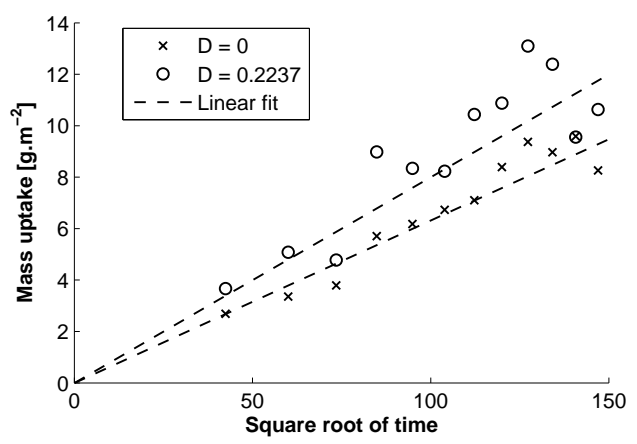

Figure 5: Profiles of mass uptake versus square root of time after a stepwise increase to $75 \% \mathrm{RH}$. The correlation coefficients of the linear approximations for $D=0$ and $D=0.22$ are respectively 0.9772 and 0.9329

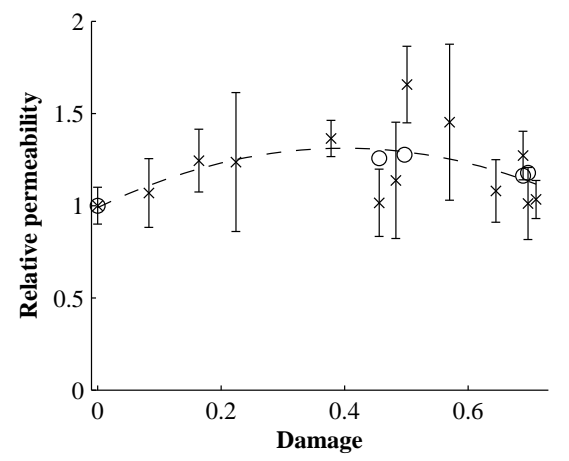

Figure 6: Average relative permeability calculated with method 1 (crosses) and method 2 (circles); the vertical lines show the standard deviation of method 1 over the series of measurements.

relative humidity. Such a mass uptake during one of the high humidity steps of the Nordtest protocol is shown for two samples in Fig. 5, along with a linear approximation.

Each time the humidity program was started, some of the scales in the chamber were occupied by non-damaged samples and the remaining scales by variously damaged samples. Each one is characterized by the ratio of its mass uptake rate to the mean mass uptake rate of the intact samples during high humidity phases of the Nordtest protocol. After $48 \mathrm{~h}$ at $50 \% \mathrm{RH}$, the protocol runs during 5 days. The value of the relative permeability is computed in all samples based on each high humidity step of $75 \% \mathrm{RH}$. The result for each sample, displayed on Fig. 6 , is the averaged permeability over these five consecutive mass uptake measurements.

According to this definition, the value of the averaged relative permeability was set to 1 for $D=0$. Each point of Fig. 6 represents one of the samples and its relative permeability presented on the graph is the mean value for all the high humidity steps it has been subjected to, in order to reduce the uncertainty of the mass measurements. The progressive wetting and drying steps of a same series of measurements may have an influence on the gap in mass uptake between intact and damaged samples, though such a trend was not captured here.

A trend can be observed, showing a potential permeability increase of up to $50 \%$ in the material before macroscopic fracture propagation. Although the uncertainties are quite high due to the high heterogeneity of the material and the characterization of damage by a single value for the entire surface of the sample, measurements have shown an increase of permeability for the earlier stages of damage, followed by a decreasing trend for higher values of $D$. A possible explanation for this behaviour is a damage process occurring in two phases: at first, a smeared pattern of microscopic cracks, perpendicular to the exposed surface, extends through the porous network, increasing its connectivity and allowing a faster vapour adsorption in the intact parts of the material. The assumption that cracks do not affect the sorption isotherm implies that they are wider than the largest pores in the virgin ma- 


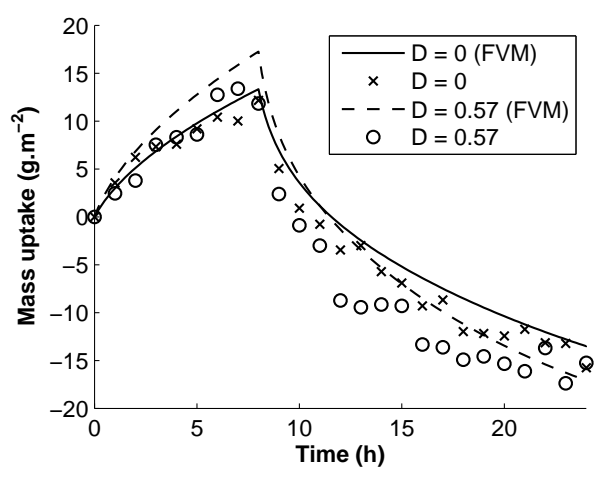

Figure 7: Mass measurements during one day of the Nordtest program and corresponding profiles computed by finite volume simulations

terial. At further loading stages, the exposed crack surface reaches a maximum value, thus no longer involving an increase in permeability. The observed subsequent decrease of permeability with increasing damage, though seemingly contradictory, can be explained by the propagation of a macroscopic fracture which causes neighboring cracks to close, thus reversing the process of increasing pore network connectivity. The crack development in this material under compression has previously been analyzed by [Elaqra et al, 2007] and showed similarities with such observations.

Since damaged have induced an increase of up to $50 \%$ in water vapour permeability, finite volume simulations were run in order to find out whether such an increase could imply a significant change in the daily moisture content fluctuations of the material submitted to the Nordtest protocol, even when considering the sorption isotherm unchanged. The transport equation for moisture transfer was implemented into a simple algorithm using 1-D finite volume method with an implicit temporal discretization. The setup of the simulations was the same as in the experiments (see Fig. 3). The humidity profile within the material was computed at each time step and summed over the domain to obtain the total water content per unit surface versus the time of the simulation. Results of these simulations are shown on Fig. 7 for a $24 \mathrm{~h}$ phase. Results are displayed as a mass uptake by unit surface.

The procedure is as follows: the mass profile of a raw sample ( $D=0$, crosses) is approximated by setting a value of $\delta_{p}$ by trial and error in the simulations. The simulations are based on the previously measured value of $\xi$ from 50 to $75 \% \mathrm{RH}$, and on an empirical value from 50 to $33 \% \mathrm{RH}$ : this lower value of $\xi$ was set so that the mass profile of the undamaged sample fitted the measurements. Therefore, eventual hysteresis effects are expected to be included by the setting of the lower value of $\xi$. Once a close fit was reached for the intact sample, the permeability was raised by $50 \%$ in order to simulate mass uptake in a damaged sample, which was compared to the measured mass profile in a sample of relative permeability of $1.5(D=0.57$, circles).

Both simulations and experiments confirmed an increase of $50 \%$ in permeability, caused by microcracking, results in a $20 \%$ higher amplitude of the daily moisture fluctuations. It must be noted that such an increase in the amplitude of fluctuations was reached although the equilibrium moisture content was considered unchanged by any amount of damage imposed to the material.

\subsubsection{Method 2: analytic resolution of the transport equation}

In addition to the study of adsorption during daily humidity cycles, the permeability was also estimated by attempting to coincide expression 13 with the measured mass uptake during a high humidity step. For this study, the climatic chamber program imposed a single $24 \mathrm{~h}$ stage of $75 \%$ relative humidity, following a supposed equilibrium state of $50 \% \mathrm{RH}$. These measurements are then used as an input for the Levenberg-Marquardt algorithm presented in Sect. 3.2.2 for an estimation attempt of $\left(\xi, h_{p}, \delta_{p}\right)$. Although the surface transfer coefficient $h_{p}$ is not a material property, its value is unknown for the present experimental configuration (ventilated climatic chamber). For the sake of this methodology, the slope of the sorption isotherm $\xi$ is considered constant in this humidity interval. This assumption is reasonable in regards to the relatively low measured value of the equilibrium moisture 
Table 2: Final computed values of the moisture permeability

\begin{tabular}{|c|ccccc|}
\hline$D$ & 0 & 0.401 & 0.497 & 0.689 & 0.697 \\
$\delta_{p}\left(\times 10^{-13}\left[\mathrm{~kg} \cdot \mathrm{Pa}^{-1} \cdot \mathrm{m}^{-1} \cdot \mathrm{s}^{-1}\right]\right)$ & 8.46 & 10.63 & 10.79 & 9.23 & 9.96 \\
\hline
\end{tabular}

content at $75 \% \mathrm{RH}$. The procedure for calculating transfer properties consists in three steps:

1. The algorithm is first run considering all three parameters as unknown.

2. The slope of the sorption isotherm $\xi$ is set to a known value, as it was previously measured in this humidity range, and the algorithm is then run considering the two remaining parameters as unknown.

3. The surface transfer coefficient was set to its average value calculated at step 2 , and the algorithm was then finally run for the calculation of the permeability $\delta_{p}$ only.

The first step, simultaneous estimation of all parameters, resulted in estimates of the moisture capacity $\xi$ far from their measured values. Such an estimation of the moisture equilibrium content based on only $24 \mathrm{~h}$ of measurements was quite inaccurate. A similar method for simultaneous approximation of the sorption isotherm and permeability based on a stepwise change in relative humidity was recently presented by [Anderberg and Wadsö, 2008]: the diffusion coefficient can be estimated within the first hours of measurements from the slope of the mass uptake curve versus square root of time. The sorption isotherm however is obtained from extrapolation of the equilibrium mass before it is reached, and an early interruption of the measurements usually leads to an inaccurate estimation. Similar results are found in this study, as trying to calculate $\xi$ from only $24 \mathrm{~h}$ of mass uptake measurements results in erroneous estimates of the sorption isotherm.

The second step corrects this fault by setting a similar value of $\xi$ for all samples, which has been measured prior to the tests. The algorithm is then used to estimate the surface transfer coefficient and the moisture permeability. This simplification is made possible by the fact that the slope of the sorption isotherm is assumed to be undisturbed by the presence of cracks and therefore is not considered a function of $D$. Five samples were used for this step of the calculation. An increase in the water vapour permeability with increasing values of $D$ could be noticed, although different values of $h_{p}$ were also obtained for all samples.

The climatic chamber in which the samples are placed is well ventilated and it is likely that the surface transfer coefficient should have similar values on all samples, as it is not a material property. The recorded values of $h_{p}$ have an average of $3.0 \times 10^{-8}\left[\mathrm{~kg} \cdot \mathrm{Pa}^{-1} \cdot \mathrm{m}^{-2} \cdot \mathrm{s}^{-1}\right]$ and a standard deviation of $6.33 \times 10^{-9}\left[\mathrm{~kg} \cdot \mathrm{Pa}^{-1} \cdot \mathrm{m}^{-2} \cdot \mathrm{s}^{-1}\right]$. Therefore, it cannot be stated that the second step of the calculations results in an accurate estimation of the permeability.

The third step of the calculations is based on this average calculated value of $h_{p}$, and is run with $\delta_{p}$ as the only unknown variable. Results of this last step are shown on Table 2 and Fig. 8. They were also included in Fig. 6 for comparison with method 1 .

These computations result in the same observations as in method 1 : the water vapour permeability increases with moderate values of $D$ and tends to decrease for higher values, but clear trend cannot be observed.

\section{Conclusion}

Two methods were presented for the estimation of the influence of mechanical damage on the water vapour permeability of fibre-reinforced mortar. The main asset of these methods is to allow for comparison of multiple samples in which the adsorption rate is measured at the same time, without a prior knowledge of the equilibrium moisture content.

The first method used in this study, based on alternative low and high humidity steps, gives a direct estimate of the behaviour of the material placed in realistic climatic hygric conditions and allows to compare the permeability of several samples which sorption isotherm is not necessarily known. The second method is directly based on the resolution of the one-dimensional moisture transport equation and also allowed the estimation of the convective mass transfer coefficient in addition to the moisture diffusivities of the samples. 


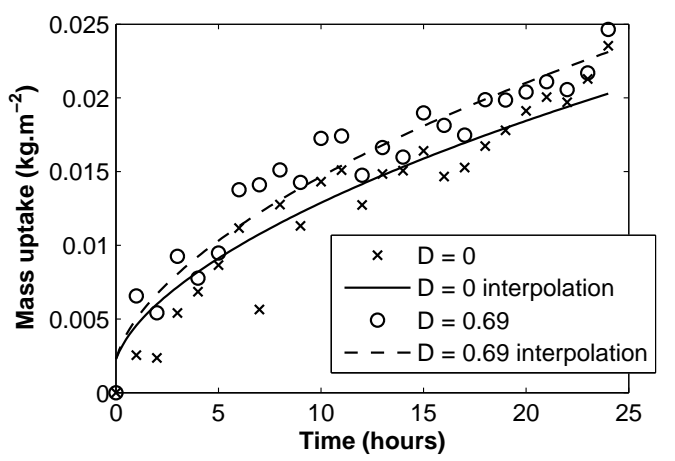

Figure 8: Mass uptake of damaged and undamaged samples during the first humidity step and fitting of the water vapour permeability

In both cases, a trend can be noticed in an increase in water vapour permeability for earlier stages of damage, followed by a plateau for medium values of $D$ and a decrease at higher values. This can be explained by the fact that the first signs of damage occur quickly once the applied stress exceeds the elastic strength of the material, causing a drop in the global tortuosity of the porous network. Further loading then seems to increase the width of these discontinuities rather than their number, which might explain a slower increase in permeability. At a further point in the loading process, the stress distribution within the material tends to concentrate on a single crack tip which, when propagating, will close neighboring microcracks. This caused previously opened paths for moisture to close and may explain the observed decreasing tendency for the vapour permeability at high values of damage. Loading stages beyond the peak strain have not been investigated here, but it is expected that the water vapour permeability is far less influenced by macroscopic fractures than the liquid conductivity, which can rise on several orders of magnitude between intact and cracked samples.

The increase rate of $\delta_{p}$ with the damage value is most likely to be influenced by the nature of the material itself, as the degradation of the elastic modulus can be caused by various forms of microstructural dislocations or, in the case of cementitious materials, of interfacial debondings. The particular formulation of mortar used in this study was specifically designed as to be less brittle as standard cement paste and to present an important diffuse damage before failure, so the observable increase in water vapour permeability might be different for other cementitious materials.

This characterization of $\delta_{p}$ as a function of a scalar damage value, combined with studies on the evolution of the liquid phase conductivity, can allow the numerical simulation of moisture transfer in existing buildings by a continuum approach, covering the entire range of relative humidities, i.e. in both liquid and gaseous phases.

Further studies on the subject are to be lead, in order to confirm the increasing trend in water vapour permeability and relate it to observations of the microstructure based on non-destructive techniques applicable to building materials, in order to dispose of quantitative measurements of the fracture network geometry to link with the measured permeability of the medium at macroscopic scale.

\section{Acknowledgements}

The results presented in this article have been obtained during the Renovbat project for the study of flow in fractured building components. Funding is provided by the Energy research cluster of the Rhône-Alpes region, which the authors would like to thank for their financial support through the project. 


\section{References}

[Aldea et al, 1999] Aldea, C.-M., Shah, S.P. and Karr, A.F.: Permeability of cracked concrete, Materials and Structures, 32, 370-376 (1999)

[Anderberg and Wadsö, 2008] Anderberg, A. and Wadsö, L.: Method for simultaneous determination of sorption isotherms and diffusivity of cement-based materials, Cement and Concrete Research, 38, 89-94 (2008)

[Baroghel-Bouny et al, 1999] Baroghel-Bouny, V., Mainguy, M., Lassabatere, T. and Coussy, O.: Characterization and identification of equilibrium and transfer moisture properties for ordinary and high-performance cementitious materials, Cement and Concrete Research, 29, 1225-1238 (1999)

[Bažant and Oh, 1983] Bažant, Z. and Oh, B.: Crack band theory for fracture of concrete, Materials and Structures, 16, 155-177 (1983)

[Bažant and Raftshol, 1982] Bažant, Z. and Raftshol, W.: Effect of cracking in drying and shrinkage specimens, Cement and Concrete Research, 12, 209-226 (1982)

[Berkowitz, 2002] Berkowitz, B.: Characterizing flow and transport in fractured geological media: A review, $A d$ vances in Water Resources, 25, 861-884 (2002)

[Carmeliet et al, 1999] Carmeliet, J., Descamps, F. and Houvenaghel, G.: A multiscale network model for simulating moisture transfer properties of porous media, Transport in Porous Media, 35, $67-88$ (1999)

[Chalencon et al, 2010] Chalencon, F., Orgéas, L., Dumont, P.J.J., Foray, G., Cavaillé, J.-Y., Maire, E. and Rolland du Roscoat, S.: Lubricated compression and X-ray microtomography to analyse the rheology of a fibrereinforced mortar, Rheologica Acta, 49, 221-235 (2010)

[Chalencon, 2010] Chalencon, F: Etude des intéractions rhéologie, fissuration et microstructure pour le développement d'un outil de formulation: application aux mortiers poreux minces fibrés dédiés à l'ITE, Ph.D. Thesis, INSA de Lyon (2010)

[Elaqra et al, 2007] Elaqra, H., Godin, N., Peix, G., R’Mili, M. and Fantozzi, G.: Damage evolution analysis in mortar during compressive loading using acoustic emission and X-ray tomography: effects of the sand/cement ratio, Cement and Concrete Research, 37, 703-713 (2007)

[Garbalinska, 2006] Garbalinska, H.: Application of $\sqrt{t}$-type, logarithmic and half-time methods in desorptive measurements of diffusivity in narrow humidity ranges, Cement and Concrete Research, 36, 1294-1303 (2006)

[Garbalinska et al, 2010] Garbalinska, H., Kowalski, S.J. and Staszak, M.: Linear and non-linear analysis of desorption processes in cement mortar, Cement and Concrete Research, 40, 752-762 (2010)

[Hagentoft 2001] Hagentoft C.E., Introduction to building physics. Studentlitteratur (2001)

[Janssen et al, 2007] Janssen, H., Blocken, B. and Carmeliet, J.: Conservative modelling of the moisture and heat transfer in building components under atmospheric excitation, International Journal of Heat and Mass Transfer, 50, 1128-1140 (2007)

[Kwiatkowski, 2009] Kwiatkowski, J.: Moisture in buildings, air-envelope interaction, Ph.D. Thesis, INSA de Lyon (2009)

[Mainguy et al, 2001] Mainguy, M., Coussy, O. and Baroghel-Bouny, V.: Role of air pressure in drying of weakly permeable materials, Journal of Engineering Mechanics, 127, 582-592 (2001)

[Marquardt, 1963] Marquardt, D.: An algorithm for least-squares estimation of nonlinear parameters, SIAM Journal of Applied Mathematics, 11, 431-441 (1963) 
[Mazars and Pijaudier-Cabot, 1996] Mazars, J. and Pijaudier-Cabot, G.: From damage to fracture mechanics and conversely: A combined approach, International Journal of Solids and Structures, 33, 3327-3342 (1996)

[Mazars and Pijaudier-Cabot, 1989] Mazars, J. and Pijaudier-Cabot, G.: Continuum damage theory - application to concrete, Journal of Engineering Mechanics, 115, 345-365 (1989)

[Pijaudier-Cabot et al, 2009] Pijaudier-Cabot, G., Dufour, F. and Choinska M.: Permeability due to the increase of damage in concrete: from diffuse to localized damage distributions, Journal of Engineering Mechanics, 135, 1022-1028 (2009)

[Polifke and Kopitz, 2005] Polifke, W. and Kopitz, J.: Wärmeübertragung, Pearson Studium, (2005)

[Réthoré et al, 2007] Réthoré, J., de Borst, R. and Abellan, M.-A., A two-scale approach for fluid flow in fractured porous media, International Journal for Numerical Methods in Engineering, 71, 780-800 (2007)

[Rode, 2003] Rode, C.: Workshop on moisture buffer capacity - summary report. Department of Civil Engineering, Technical University of Denmark, Lyngby (2003)

[Roels et al, 2006] Roels, S., Moonen, P., Proft, K. D. and Carmeliet, J.: A coupled discrete-continuum approach to simulate moisture effects on damage processes in porous materialsn, Computer Methods in Applied Mechanics and Engineering, 195, 7139-7153 (2006)

[Scheffler and Plagge, 2010] Scheffler, G.A. and Plagge, R.: A whole range hygric material model: Modelling liquid and vapour transport properties in porous media, International Journal of Heat Mass Transfer, 53, 286-296 (2010)

[Wang et al] Wang, K., Jansen, D.C., Shah, S.P. and Karr, A.F: Permeability study of cracked concrete, Cement and Concrete Research, 27, 381-393 (1997)

[Xu et al, 1997] Xu, K.,Daian, J.-F. and Quenard, D.: Multiscale structures to describe porous media - part 1: theoretical background and invasion by fluids, Transport in Porous Media, 26, 51-73 (1997)

[Xu et al, 1997] Xu, K.,Daian, J.-F. and Quenard, D.: Multiscale structures to describe porous media - part 2: transport properties and application to test materials, Transport in Porous Media, 26, 319-338 (1997) 